\title{
Unleashing the power of human genetic variation knowledge: New Zealand stakeholder perspectives
}

\author{
Yulong Gu, $M S^{I}$, James Roy Warren, $P h D^{1,2}$, and Karen Jean Day, PhD
}

\begin{abstract}
Purpose: This study aimed to characterize the challenges in using genetic information in health care and to identify opportunities for improvement. Methods: Taking a grounded theory approach, semistructured interviews were conducted with 48 participants to collect multiple stakeholder perspectives on genetic services in New Zealand. Results: Three themes emerged from the data: (1) four service delivery models were identified in operation, including both those expected models involving genetic counselors and variations that do not route through the formal genetic service program; (2) multiple barriers to sharing and using genetic information were perceived, including technological, organizational, institutional, legal, ethical, and social issues; and (3) impediments to wider use of genetic testing technology, including variable understanding of genetic test utilities among clinicians and the limited capacity of clinical genetic services. Targeting these problems, information technologies and knowledge management tools have the potential to support key tasks in genetic services delivery, improve knowledge processes, and enhance knowledge networks. Conclusion: Because of the effect of issues in genetic information and knowledge management, the potential of human genetic variation knowledge to enhance health care delivery has been put on a "leash." Genet Med 2011:13(1):26-38.
\end{abstract}

Key Words: genetic services, genetic testing, grounded theory, information technology, knowledge management

$\mathrm{O}$ ur understanding of the genetic component of diseases has increased significantly, especially since the completion of human genome sequencing in 2003. ${ }^{1}$ Applying human genetic variation knowledge, where associations between genetic variations and conditions are identified, clinical genetic services provide genetic testing and results interpretation for patients and families with existing or suspected genetic disorders. ${ }^{2}$ The US National Library of Medicine defines genetic testing as a type of medical test that identifies changes in chromosomes, genes, or proteins. ${ }^{3}$ Medical genetic testing referrals can be made by clinical geneticists, genetic counselors, and primary or specialty care providers. ${ }^{2}$

It is surprising that health care has not been transformed by the revolution of (molecular) genetic testing, as predicted by many. ${ }^{4-8}$ Genetic test results, along with the information gained from family history and physical examination, can be used to diagnose medical conditions, to assist in reproductive decision making, to predict future risks to health, and to suggest treatment in patient care. 9,10 The use of genetic information and its

From the ${ }^{1}$ Department of Computer Science, and ${ }^{2}$ School of Population Health, University of Auckland, Auckland, New Zealand.

Yulong Gu, Department of Computer Science-Tamaki, University of Auckland, Private Bag 92019, Auckland, New Zealand. E-mail: ygu029@cs.auckland. ac.nz.

Disclosure: The authors declare no conflict of interest.

Submitted for publication June 28, 2010.

Accepted for publication August 25, 2010

Published online ahead of print November 9, 2010.

DOI: 10.1097/GIM.0b013e3181f9648a ability to predict susceptibility to disease or guide proactive care suggest the potential of personalized medicine to transform health care systems. ${ }^{11}$ However, there is ongoing debate around the utility of medical genetic testing and the scope for genetic intervention. ${ }^{12}$ The technology of genetic testing is complicated and has limitations. There is a poor understanding of the utility and clinical validity of genetic tests outside of the specialist setting and notably a lack of practical knowledge among general practitioners (aka family physicians or GPs). ${ }^{13}$ This knowledge deficit issue was also reported in the United States, ${ }^{14}$ the United Kingdom, ${ }^{15,16}$ and Australia. ${ }^{17}$ In addition, there is a lack of principles or strategies for handling genetic information in health systems. Adding to this complexity is the sensitivity of medical genetic test results, which presents challenges for managing and sharing these data among care givers to improve health and for data dissemination to facilitate knowledge management (KM).

Given these challenges, this study examined the perspectives of stakeholders in New Zealand on the use of genetic information in health care. This study aimed to analyze the range of practice in use of information relating to genetic services and to uncover perceived gaps and opportunities within the existing information technology (IT) environment. This investigation provides groundwork for refining KM processes to meet the needs of people using and working in genetic services delivery by understanding:

How does the New Zealand genetic services system work? How should it work in the future?

What are the barriers to sharing and using genetic information?

Why is genetic testing technology not more widely used in clinical practice?

What are the KM requirements from genetic services stakeholders?

What are their experiences, expectations, and concerns?

With no assumed answer to these questions, we developed our grounded theory on systematically collected data, including interview transcripts, literature, and organizational documents, as well as our notes during the research.

\section{METHODS}

Medical genetics is a relatively new science with new and sometimes conflicting knowledge emerging at a rapidly increasing rate. ${ }^{15}$ Therefore, we decided to use grounded theory to tease out information management and KM challenges that are specific to medical genetic testing and the associated clinical care. Grounded theory is an inductive methodology to generate theories through a rigorous research process leading to the emergence of conceptual categories; and these concepts as categories are related to each other as a theoretical explanation of the actions that continually resolve the main concern of the participants in a substantive area. ${ }^{18,19}$ We took the classic grounded theory approach to develop an understanding of the 
New Zealand genetic services system and of the stakeholder perspectives concerning genetic information and KM issues.

A semistructured interview schedule was developed for the purpose of gaining ethics approval (which was granted by the University of Auckland Human Participants Ethics Committee). This schedule was adjusted as the interviews developed. Questions on the schedule were used as prompts for interviewees to talk about topics, themes, ideas, and concepts that they felt were key to the research topic. These questions include "What do you think would be the appropriate means of distribution for genetic testing results?" "What changes would you like to see in handling and managing genetic information?" and "What are the current and potential challenges from your point of view?" Convenience sampling was used to start collecting interviews with patients, doctors, genetic counselors, and genetic testing laboratory scientists. Snowballing occurred as we conducted interviews and asked interviewees for suggestions about whom to include in the study. Although the theory was emerging, we used the method of theoretical sampling to gain insights from particular groups of stakeholders, e.g., medical specialists, patients, and health insurance providers.

Data analysis was conducted as interviews proceeded until we reached data saturation (i.e., we were hearing repeated opinions and few new topics). The interview transcripts were processed by multiple coding (line-by-line open coding in QSR NVIVO software, ${ }^{20}$ axial coding, and selective coding) and constant comparative analysis, as suggested by Strauss and Corbin. ${ }^{21}$ The elements of our grounded theory, including the data categories, properties of category, and themes, were identified during this process. We applied the interpretivist paradigm, especially the hermeneutics principle, ${ }^{22}$ to interpret multiple perspectives. We also took a general inductive approach ${ }^{23}$ to summarize the data categories from participant comments and to identify relationships among these categories and their properties. Three themes emerged from these relationships, which led to the development of our final theory. In addition, the analytic comparison strategy ${ }^{24}$ with a critical view ${ }^{25}$ was adopted to identify differences among all the data gathered in this study, including interview data incidents, multiple perspectives, actual operations, and organizational policies or literature.

The interview findings that emerged from the above process were triangulated and verified by cross examination from multiple data sources. These data include organizational documents, guiding policies, literature, and our notes. Additional triangulation was achieved using the semantic space modeling technique by Burgess and Lund, ${ }^{26}$ hyperspace analog to language (HAL). This allowed us to test our grounded theory for bias, including researcher subjectivity as mentioned by Klein and Myers ${ }^{27}$ and Kock. ${ }^{28}$ HAL modeling generates a term-term matrix; and term frequencies are calculated according to the degree terms cooccur within a sliding window. ${ }^{29}$ We moved a window 10 words in width across the interview transcripts (after discarding stop words and the interviewer's questions), and wherever two words occurred within the window, the value at their intersection in the HAL matrix was incremented; thus, the text was converted to a high-dimensional semantic space, as described by our colleagues in applying HAL for breast cancer web pages analysis. ${ }^{30}$

We used HAL matrixes at two points in the data analysis. First, after open coding on the first 10 interview transcripts, we calculated the 40 terms whose HAL vectors had the largest sum based on the interview data (basically, frequent terms). These 40 terms were then used to identify meaningful markers to inform summarization of data categories and properties from the transcripts. The second use of HAL calculations was to triangulate our final results against quantitative matrixes of HAL. We generated two HAL matrixes from all interview transcripts and compared the features of these matrixes to our theory. In particular, we verified that the concepts in our qualitatively developed grounded theory fell into the most frequently cooccurring pairs of terms according to HAL.

Analysis of IT and KM opportunities was based on participant comments and requirements, also based on our review of literature in health informatics, bioinformatics, KM, regulations, and policies. The collective opinions, triangulated by literature, were summarized to derive a set of promising technologies to support genetic services delivery in the future.

\section{RESULTS}

This study collected multiple stakeholders' perspectives on New Zealand genetic services by semistructured interviews with 48 participants in 37 sessions. (Some discussions were attended by multiple participants, who work in the same laboratory or regional office.) As listed in Table 1, we involved a total of 48 research participants representing nine categories of stakeholders in the clinical genetic services system (where often an individual had more than one role).

\section{Data categories}

General inductive analysis was conducted based on the open coding results of the first 10 transcripts. These 10 sessions collected perspectives from patient/family member, researcher, governance, health care provider, genetic counseling professional, genetic testing laboratory, and IT professional. After open coding, we calculated a HAL $40 \times 40$ matrix on these 10 transcripts to characterize the context of the 40 most prominent terms; that is, those whose vectors have the largest sum in the HAL matrix. (Given the derivation of HAL, these should be

Table 1 Roles of the research participants in the New Zealand genetic services system

Participant's role
Patient and family member
Healthcare provider (including three GPs and five medical
specialists)
Genetic counseling professional (including three clinical
geneticists and eight genetic counselors)
Genetic testing laboratory scientist
Governance (including two managers at the New Zealand
Ministry of Health and an advisor at the Privacy
Commission)
Total $^{a}$ 8 8

Health IT professionals

Researcher in health and/or health IT

Director of a health institution that provides genetic services, e.g., hospitals, general medical laboratories, genetic testing laboratories, and regional genetic services

Clinical advisor at an indirect health service, e.g., health insurance companies, health IT vendors, and IT services at district health boards (DHBs)

${ }^{a}$ Some participants have more than one role in the system, for instance as clinician and director. 
Table 2 Highlighting the prominent terms by HAL calculation

\begin{tabular}{ll}
\hline $\begin{array}{l}\text { The } 40 \text { top-scored HAL terms in } \\
\text { first } 10 \text { transcripts }\end{array}$ & $\begin{array}{c}19 \text { meaningful markers } \\
\text { in the } 40\end{array}$ \\
\hline $\begin{array}{l}\text { Genetic, information, people, family, } \\
\text { testing, test, cancer, result, patient, }\end{array}$ & $\begin{array}{c}\text { People, patient, person, } \\
\text { patients, }\end{array}$ \\
clinical, mutation, time, gene, & family, families, \\
breast, diagnosis, services, & cancer, result, results, \\
medical, data, families, insurance, & diagnosis, insurance, \\
person, risk, consent, work, & risk, consent, \\
service, condition, important, & condition, disease, \\
patients, disease, genetics, health, & report, research, lab, \\
find, report, research, results, lab, & database. \\
tests, database, part, understand. &
\end{tabular}

essentially the 40 most frequent words in the interview corpus.) Aiming to identify the most insightful responses that indicate problematic issues for further exploration, we chose 19 words out of these 40 as meaningful markers. The remaining 21 words were discarded because they were:

1. Stop words, e.g., "time" and "part."

2. Merely components of the overall topics of the interviews, e.g., "genetic," "information," and "testing."

3. Obviously subsumed in the concept of another term, e.g., "breast" as in "breast cancer."

Having identified 19 terms that would be informative for producing data categories or properties, we grouped these 19 words, as per Table 2 .

On the basis of the semantically meaningful markers in Table 2 , we highlighted the 19 terms with colors in the interview transcripts. Assisted by these colored markers, we read the transcripts again, compared among data incidents, and reexamined the open codes to identify data categories and properties. Manifestation of the data categories was also helped by an exercise of summarizing and conceptualizing participant comments into around 180 brief statements, printing and cutting these statements into paper slips, then grouping the slips into categories manually. Five categories of topics were discussed by the participants that are relevant to the use of information during genetic services delivery processes. These data categories were enriched with more incidences and confirmed later to be the most frequent issues by the rest of interviews as we approached data saturation.

\section{Category 1: The clinical utility of genetic testing}

One frequent topic in the conversations was whether genetic testing is useful in health care. The interviews collected mixed perceptions of genetic test utilities. Participants' understanding of the clinical utility of genetic tests varied according to their roles, experience, and knowledge. Genetic testing technology is believed to have great complexity in some cases. One complexity, particularly as a barrier to health care providers, is the lack of information about test prevalence and penetrance. Nonetheless, genetic testing was reported to have the potential to produce critical information for:

- Diagnosis, e.g., the human ether-à-go-go-related gene test for type 2 long QT syndrome diagnosis.

- Treatment, e.g., the human epidermal growth factor receptor 2 test in choosing breast cancer therapy.
- Predicting cancer occurrence risk or assessing cancer recurrence risk, therefore directing surveillance and preventive management such as mastectomy, or treatment, e.g., the $B R C A 1, B R C A 2$ tests, and the multigene expression array test using MammaPrint ${ }^{31}$ or Oncotype. ${ }^{32}$

- Checking carrier status and pregnancy planning, e.g., Delta F508 mutation detection for cystic fibrosis, which may encourage a couple to pursue preimplantation genetic diagnosis with in vitro fertilization.

- Lifestyle planning, e.g., the huntingtin gene presymptomatic test for Huntington disease.

- Avoiding adverse drug reactions, e.g., in prescribing warfarin and azathioprine.

Despite all the above clinical utilities identified by our participants, the general view found in our study was that genetic testing in primary care is considered futuristic. In contrast, there was a sense that it would eventually become part of everyday health care. The goal of clinical genetic services, according to genetic counselors, is to improve the health of patients and families, not only in acute care but also in continuous health management for future generations.

\section{Category 2: Information requirements}

Based on a basic genetic services delivery model - the PatientDoctor-Counselor Model, we summarized the genetic information use and access requirements in Figure 1. The main steps in this formal genetic service program are (1) a patient presents with symptoms or concerns of a genetic disorder at a doctor's office (GP or specialist). Based on relevant knowledge and experience, the doctor might believe a genetic test to be useful for diagnosis, prognosis, or treatment. (2) Therefore, the doctor makes a referral to a regional genetic service office, where genetic counselors evaluate and sort referrals in a triage process. (3) In a face-to-face meeting with the patient, a genetic counselor (or a clinical geneticist, if a diagnosis is not given by the referring doctor) assesses the disease risk; and if a genetic test is relevant and available, they counsel the patient about testing and explain the possible results and their implications, e.g., implications for insurance. If the patient consents to the test, this is then arranged by the genetic counselor. (4) The patient needs to go to a general medical laboratory for sample collection (often a blood sample), which is sent to an accredited genetic testing laboratory according to a waiting list in the regional genetic service office. (5) Laboratory scientists perform the test and record any detected abnormality and its interpretation in a laboratory report. (6) Based on this report, the genetic counselor writes an explanatory letter suggesting surveillance recommendation and/or management intervention. After counseling the patient about this result, the genetic counselor stores the doctor referral, family history, family tree, laboratory report, and explanatory letter into a family folder. This folder will be kept indefinitely in the genetic service office as a reference for family members and future generations. On patient consent, copies of the laboratory report and explanatory letter can be sent to health care providers.

When a patient is routed through the genetic services system by the Patient-Doctor-Counselor Model, documentation is kept as the patient's medical record at the doctor's office, doctor's written referral to the regional genetic service office, genetic test report by a testing laboratory, explanatory letter given to patient by genetic counselor, and the family folder in genetic service office. Beyond the key parties inside this model (the patient, doctor, genetic counselor, and laboratory scientist), external use of the information may be required by additional parties. These include family members of the patient, health care profession- 


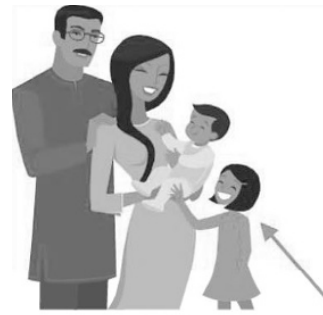

An individual's genetic test results may have implications for other family members.

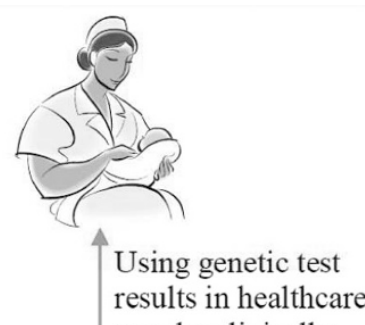

may be clinically significant, e.g. for midwives, obstetrician, oncologists, surgeons, etc.

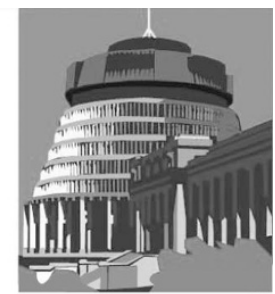

Ministry of Health needs statistical info on genetic tests to govern the services.

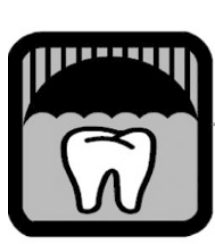

Accessing

genetic test results may be of interest to third parties, such as health insurance companies.

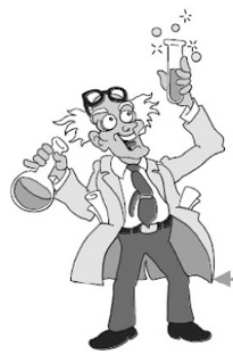

The results produced in testing labs are significant data for (clinical) genetics researchers to advance human genetics science.

1) A patient goes to see a GP. 2 2) GP refers the patient to a 3) Genetic counsellor genetic genetic

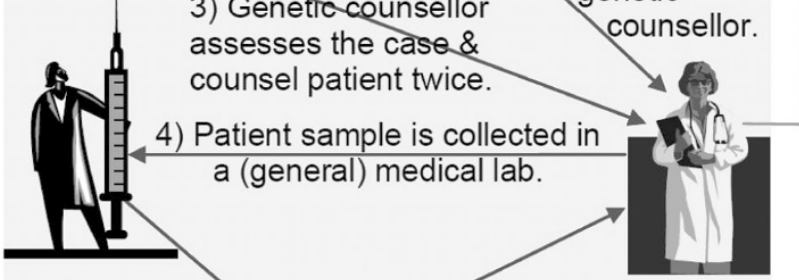

5) Testing data are analysed by genetic testing lab scientists

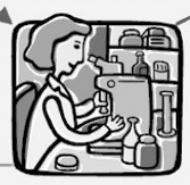

6) Test results are reported back to genetic counsellor, who will discuss results with the patient ONLY. Patient-Doctor-Counsellor Model

Fig. 1. Information requirements based on the Patient-Doctor-Counselor Model.

als, genetic services governance authorities, health insurance companies, and genetics researchers. Given all these requests, a regional genetic service director concluded that the current data distribution principles for genetic test results are (a) because a patient wishes it to be and (b) because it is going to benefit the patient in some way.

In Figure 1, the data flow inside the Patient-Doctor-Counselor Model and those extending from it demonstrate the information needs of multiple stakeholders involved in delivering high-quality health care. Many participants acknowledged the gap between the current incoherent data management practices and an ideal system environment that supports information flows, KM (especially research data dissemination), and stakeholder communication. Opportunities present themselves to improve the storage and sharing of genetic test results and genetic testing-related information. It was understood by all interviewees that the provision of clinical genetic services requires primary, secondary, and tertiary health systems to interact with regional genetic service offices and laboratory services. However, concerns were raised regarding the inconsistency, inefficiency, and nonstandardization in the current documentation style and information transfer practices. The isolated storage of genetic test results may jeopardize the quality of acute care, disease management, and health care planning. It was also recognized that there is a lack of resources and standards for genetic data dissemination, especially in terms of contributing to global genetic knowledge bases and distributing results in families. A genetic counselor mentioned:

It would be nice to know-why people haven't come forward for testing sometimes, the family. But we've got no way of knowing that. That's invading their privacy. We can't go contacting them.

With this question on the efficacy of family communication, we went out seeking more patients to interview and discovered a range of issues entangled with the distribution of genetic test results in families. It is often a burden on the patients themselves, as they often feel guilty and are themselves critically ill; there is a lack of information support for patients and family members; and there are complex implications in terms of ethical dilemmas and privacy challenges. Communication can potentially be more effective in the Māori cultural context, where a genetic counselor may discuss results at a hui (formal meeting) of the whänau (extended family) but alternatively where elders may decide against dissemination of the results.

\section{Category 3: Regulation and governance effectiveness}

Clinicians participating in genetic services delivery felt that they were at times inventing principles around genetic information use and disclosure. Many of our participants believed that 
genetic services, impaired by policy gaps, are not serving the population well. A privacy commission advisor raised the questions of:

How do the normal ethical constraints, about doing no harm and providing information when necessary to protect somebody's life or health, apply when it's not just information about the person taking the test? ... How does the generality of genetic information, inasmuch as it relates to multiple people at a time, interact with the ethical and legal constraints on disclosure?

Additional ethical implications of genetic test results add even more sensitivity into the information. For instance, reproductive choices are affected because the mutations contained in a sperm or egg (germline mutations) may be passed to offspring. 8,33 Another ethical and legal challenge is the access right by health insurance providers who can exclude coverage related to a mutation. In practice, the genetic information disclosure to family members and insurers (both as a third party) follows the Human Genetics Society of Australasia's best practice guideline on consent process, which aims to protect patients' right to confidentiality and privacy. However, New Zealand has no equivalent legislation to the US Genetic Information Nondiscrimination Act $^{34}$ to protect patients against genetic discrimination.

\section{Category 4: Clinicians' knowledge about genetic testing}

During the interviews, it also became clear that the clinicians' attitudes toward clinical genetic services are largely influenced by the clinical usefulness and the health outcomes of genetic testing. Even the clinicians who already used genetic testing in patient care still need clear protocols and guidelines to support the process. Information support is needed to involve the rest of health care providers in the genetic service delivery system, such as information about clinical utility and availability of genetic tests, about disease risk assessment protocols, referral processes, and clinical guidelines. A GP related: "Genetic information would perhaps apply more specifically to genetic markers, and we might want to see a few more instances before a clearer picture emerges." Genetics education for health professionals has been recognized as a critical factor to develop genetic services capacity in Scotland, ${ }^{35}$ Australia, ${ }^{36}$ and New Zealand, ${ }^{13}$ especially considering the rapidly developing genetic testing technologies.

\section{Category 5: Genetic testing technologies and (private) laboratory resources}

In New Zealand, a number of genetic tests for cancers and some rare disorders are using highly specialized and accredited overseas laboratories, e.g., $B R C A 1$ and $B R C A 2$ testing in Australia. New Zealand laboratories also accept test requests from overseas, e.g., Delta F508 mutation detection for cystic fibrosis. Also available to patients are the private "direct to consumer" genetic testing services. ${ }^{37}$ In terms of the genetic testing technology itself, several participants mentioned the next generation sequencing technology. ${ }^{38}$ Some believed that within 5 or 10 years, this technology would enable the sequencing of any patient's whole genome. Managing the huge volumes of the sensitive data produced by these new sequencing technologies would then present a novel and fundamental challenge for genetic information management in the genetic services delivery system. In addition, our study has identified three barriers (as three data themes in the next section) that are inhibiting this health service to deliver better health care outcomes.

\section{Data themes}

Throughout the open coding, axial coding, and selective coding on participant comments, we constantly compared the emerging messages with the five initial data categories (based on the first 10 interviews). Three themes emerged by defining the relationships among all interview data categories when we reached data saturation, which was achieved with the theoretical sampling technique. For instance, we sampled medical specialists, patients, and insurers aiming to use their perspectives to check any possible contradiction of understandings. Using the general inductive analysis method, we clustered all 110 open codes from the whole interview data set into three data themes, as numbered in Figure 2.

The data analysis focused on discovering the diverse degrees of understandings among similar and different stakeholder groups, the relationships among discussed topics, and the conflicting opinions on approaches to solving problems. Three themes emerged from this analysis and were validated by data triangulation with literature and our notes, as well as by applying HAL modeling technique again. The resulting themes are as follows:

1. The multiple operation models in the New Zealand genetic services system

2. The inhibited genetic data sharing.

3. The barriers to wider use of genetic information.

\section{Theme 1: Four genetic services delivery models are available to New Zealand patients}

Contrary to our expectations derived from the structure and service access processes in the New Zealand health system, three variations of the Patient-Doctor-Counselor Model were identified. They are the Patient-Doctor-Lab Model, the Patient-Counselor-Lab Model, and the PatientLab (Commercial) Model. Each of these four available models involves different parties, varied processes, and data flows, as compared in Figure 3. They operate with various doctor referral processes and nonstandardized documentation styles, see also Ref. 39. With these differences, the communication of genetic test results and their implications is not managed in a systematic manner. Inadequate information is available to stakeholders; incomplete documentation is recorded in some models; and there is little support for communication or result dissemination.

One noticeable issue with the Patient-Doctor-Counselor Model (Fig. 3.1) is that the referring doctors do not receive the test results in a timely fashion. A medical specialist described the experience: "You just don't know what's happening, you send off a request and it goes into a long black hole." The Patient-Doctor-Lab Model (Fig. 3.2) demonstrates that clinicians can order some tests directly from a genetic testing laboratory without routing through genetic services, e.g., the hemochromatosis gene test for hemochromatosis diagnosis. However, the doctor's note does not keep a comprehensive family folder as in genetic service offices. In the Patient-Counselor-Lab Model (Fig. 3.3), if a pathogenic mutation has been identified in a family, family members may bypass the doctor and contact genetic counselors directly; subsequently, their doctors may not be informed about the test result. The Patient-Lab (Commercial) Model (Fig. 3.4) is made possible by private companies that offer "direct to consumer" genetic testing, such as 23 andMe. ${ }^{40}$ 


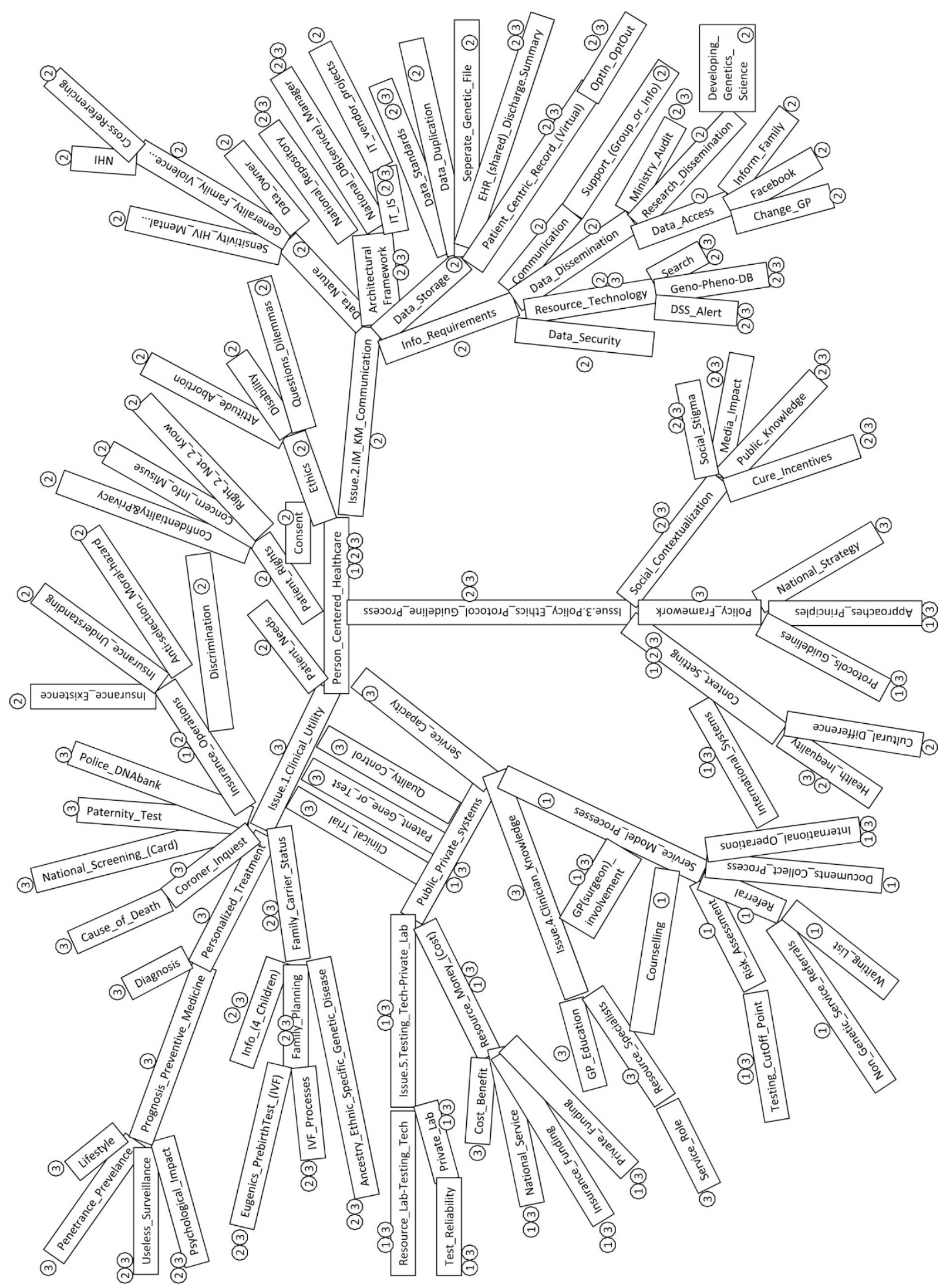

Fig. 2. The emergent themes of the study. 


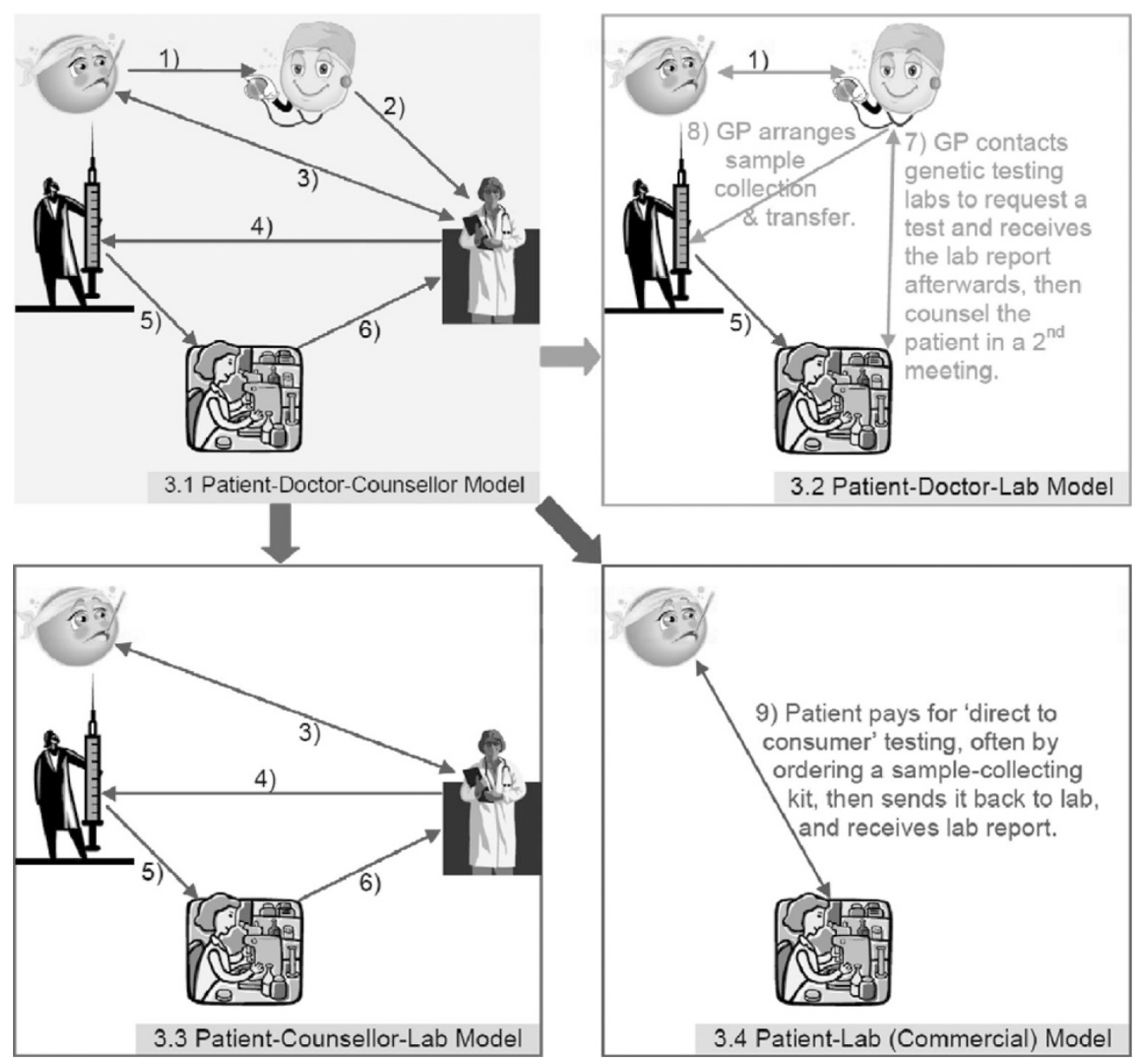

Fig. 3. Four genetic services delivery models are available to New Zealand patients.

Most participants in our study perceived this last model as dangerous because commercial services may use unaccredited laboratories, may not offer proper counseling, and may not comply with existing guidelines.

\section{Theme 2: Genetic data sharing is inhibited}

Theme 2 emerged from the interview data relating to genetic information dissemination. We found that the distribution of genetic test results was limited in both individual sharing (e.g., family communication of the clinical implications of a pathogenic mutation) and aggregated sharing (e.g., research data dissemination to global knowledge bases). Several issues were perceived as barriers to sharing genetic information for health care purposes, including technological, organizational, institutional, legal, ethical, and social issues. Our study identified a lack of IT support for information circulation (e.g., with outof-date reporting systems in genetic testing laboratories), several ethical and legal challenges (e.g., an obvious question emerged: now that I've got the result, what can I do with it and what must I do with it?), complex patient needs versus patient rights (e.g., the burden and difficulty of family communication), and gaps in New Zealand policies (e.g., to protect patients against genetic information misuse, discrimination, and health inequality).

A significant factor inhibiting genetic data sharing is the sensitivity levels of genetic information. For instance, a test result that indicates a genetic predisposition for adverse drug reaction to warfarin is not as sensitive as that of a carrier status result that is associated with a severe disability or a significantly shortened life expectancy. This sensitivity level seemed to have strong indications for a patient's intent to share the information or not, e.g., with their care givers and family members. It also determines genetic service providers' protocols for protecting the family's privacy. However, the experience from managing traditional sensitive medical data, such as HIV status, has proven that the development of a legislative framework can lead to a rapid change of attitude toward the data sharing. For instance, the Human Rights Act prohibits discrimination against people with HIV. ${ }^{41,42}$ As a result, HIV test results have become more mainstream medical data and hospitals no longer deliberately suppress them. Similarly, until a legislative framework is established to support and protect individuals with genetic test results, some genetic information may remain sensitive and, therefore, not shared for health care or research purpose.

Given the challenges in genetic data sharing, divided attitudes were noted among participants toward whether genomics is something new and requiring a different approach in the health care delivery. Some had concerns about the possible information misuse when keeping genetic information, which they considered to be a unique type of data, in an electronic form permanently. Others acknowledged the shared nature and 


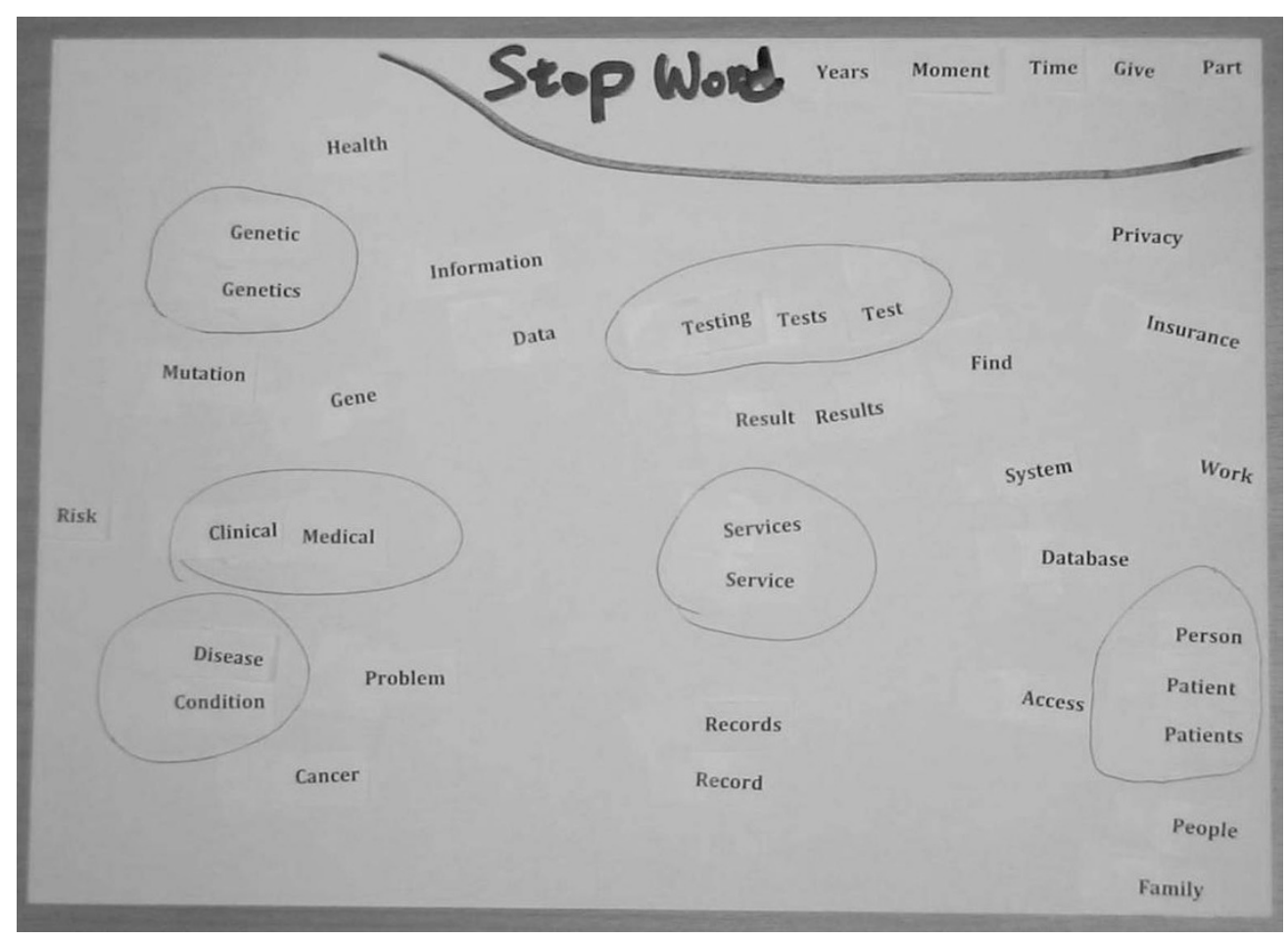

Fig. 4. Thematic clusters of high-frequency concepts in the interview data.

prognostic power of genetic data but proposed to store and use them as any other medical data. In addition to the data sharing barriers, we have identified other barriers to a wider use of genetic testing.

\section{Theme 3: There are barriers to a wider use of genetic testing}

The impediments to wider use of genetic testing technology, beyond inhibition about the sharing of genetic test results per se, include the variable understanding of genetic test utilities, particularly in clinicians, and the inadequate capacity of clinical genetic services. The uncertain clinical utility of genetic tests and the associated gaps in clinician knowledge present a major barrier to involving health care providers in genetic services delivery. Our study participants reported scarcity of information available to clinicians regarding (1) clear evidence for test utilities, (2) protocols for choosing among multiple test pathways, and (3) clinical guidelines for deciding among many possible patient management actions based on the test results, see also Ref. 43. Clinicians seem to help in identifying the clinical utility of genetic tests and developing core competencies. They need support with the key tasks in delivering genetic services, such as assessing disease risks, identifying the benefits and availability of genetic tests, streamlining referral processes, and managing patients according to test results.

Another barrier in genetic services delivery is the service capacity, in terms of counseling workforce, laboratory facilities, and other resources such as funding. The World Health Organization recommended one clinical geneticist and two genetic counselors per 500,000 population; thus, the 4 million New Zealanders need eight clinical geneticists and 16 genetic counselors. ${ }^{44}$ We have roughly the equivalent of five full-time clinical geneticists and nine full-time genetic counselors, which is just above half the number recommended by the World Health
Organization. Laboratory facilities in New Zealand are also limited. A range of genetic tests can be done by laboratories in New Zealand, but a number of routine genetic tests and some rare disorders tests are conducted overseas, including BRCA1 and BRCA2 testing in Australia. Funding problems lead to rationed prioritization processes and waiting lists, with some people waiting as long as 3 years for nonurgent tests.

In the mean time, international health systems are starting to share genetic test results to deliver quality care. This is because internationally scattered family members share genetic characteristics. Both permanent and, increasingly with internationalization of the workforce, for a term of a few years, flow through is very common in many countries. For instance, at the time of the 1996 New Zealand Census, there were 605,019 people living in New Zealand who were born overseas, making up $17.5 \%$ of the resident population. ${ }^{45}$ The New Zealand population remains highly mobile, with 82,700 permanent or longterm arrivals and 72,600 departures in the 2006/2007 financial year. ${ }^{46}$ Considering these flow throughs and the mobile population, the provision of quality health care requires international health systems to interact with each other. This demands support for communication and cooperation among genetics centers, testing laboratories, care facilities, and funding agencies.

\section{Grounded theory: Emergence and verification}

Each of the above three data themes was underpinned by the responses of a diverse range of stakeholders. In addition, each theme represented a distinct set of forces that impair the realization of the full potential of genetics knowledge in health delivery. The amelioration of these impediments should be the cornerstone of any strategy to achieve higher utilization, and thus maximum benefit, from medical genetics. Therefore, we summarized the findings of this study into a theory of unleashing the power of human genetic variation knowledge: to realize 
the full potential of human genetic variation knowledge for better health care outcomes, we need to overcome the challenges of multiple service models, the inhibitors for genetic data sharing, and the barriers to wider use of genetic testing.

The HAL technique was applied to verify the above theory. A $40 \times 40$ HAL matrix was computed, and 40 terms were identified with the highest HAL score. Examining these 40 terms, we created the thematic clusters of concepts from our interview data, as shown in Figure 4.

Examining Figure 4, these HAL-identified frequent concepts have all been presented in the data categories and themes from qualitative analysis. According to this result, we (a) added five more stop words (as identified in the right top corner of Fig. 4), (b) combined terms with common stems or meaning into domain concepts (as circled in the Fig. 4), and then (c) recomputed HAL and extracted a $10 \times 10$ term-term matrix. The 40 most frequently occurring term-term pairs were extracted from this matrix and compared with our data themes and final theory. Table 3 divides these pairs into three groups - (1) term-term pairs indicating a close relationship between two concepts, (2) term-term pairs forming one concept, and (3) the concepts that frequently cooccur with themselves.

Comparing our final theory with Table 3 shows that our results based on the qualitative grounded theory approach have covered the most discussed domain concepts in our interviews. Moreover, a couple of interesting pairs caught our attention from the HAL calculation, including cancer_breast and insurance_insurance. Although we did not produce a specific theory on breast cancer, its appearance in Table 3 does not contradict our results, because breast cancer was a dominant case in the interviews. It was frequently referred to by health care professionals, genetic counselors, laboratory scientists, patients, and

Table 3 HAL verification: 40 most frequently cooccurring pairs of terms

\begin{tabular}{lll}
\hline Related two concepts & \multicolumn{1}{c}{ One concept } & \multicolumn{1}{c}{ Intensified concept } \\
\hline Information_people & Test_genetic & Test_test \\
Test_people & Information_genetic & Information_information \\
Genetic_people & Genetic_service & Genetic_genetic \\
Information_patient & Cancer_breast & People_people \\
Test_family & Family_history & Patient_patient \\
Test_patient & Information_medical & Family_family \\
Genetic_patient & Test_results & Insurance_insurance \\
Test_information & Test_result & Medical_medical \\
Genetic_medical & Test_gene & Cancer_cancer \\
Information_family & Genetic_condition & Record_record \\
Information_health & Information_access & Condition_condition \\
Patient_family & Health_service & \\
People_family & & \\
Test_medical & & \\
Test_condition & & \\
Genetic_family & & \\
Family_cancer & & \\
\hline
\end{tabular}

insurance providers. The intensified concept of "insurance" by its frequent cooccurrence with itself does indicate the participants' strong interest in commenting about insurance and insurance related issues. We discussed its relevance in our second theme regarding genetic data sharing and regarding the ethical and legal implications of genetic information. The relationships between the concepts that surfaced from the HAL matrix validate our qualitative analysis in that they fall within the scope of our data themes.

\section{IT/KM opportunities}

Participant comments in our study identified several opportunities for refining and better supporting the existing health information practices. These opportunities include to store and share genetic test results with consideration on the complexity of genetic data and their implications for the health of individuals and their relatives and descendants, to standardize the specialized genetic concepts and terminology, to share genetic data beyond the scope of everyday clinical care (e.g., for KM purpose), to organize information in decision support systems (DSS), and to realize the vision for electronic referral systems (e-Referrals) as put forth in the New Zealand Health Information Strategy Advisory Committee. Health Information Strategy Advisory Committee pointed out that future e-Referrals should facilitate referrals tracking, electronic status reports, acknowledging referral receipts, and generating alerts if service level timeframes are not met. ${ }^{47}$ Technologies such as e-Referrals, DSS, and electronic health record (EHR) or personal health record (PHR) systems may have the potential to support key tasks in the clinical genetic services delivery. Key technologies and their functions are summarized in Table 4.

The ultimate goal of IT/KM tools (as listed in Table 4) is to facilitate the processes of knowledge creation, integration, sharing, and reuse by supporting the flow of genetic data that are represented in structure and are interpretable through all stakeholders. For instance, knowledge needs to be transferred from genetics science to clinical practice (supported by tools such as DSS for clinicians during key tasks in service delivery). It should also feed back to the body of the human genetic variation knowledge through data sharing. PHR may facilitate the sharing of test results and risk information among family members. e-Referrals have the potential to share test status updates across health sectors. Social networking tools, such as online patient support groups, are useful to share knowledge and experiences in the community. Gene ontologies and submission tools (with appropriate deidentification approach) are essential in data dissemination to knowledge bases. Moreover, knowledge networks among clinicians, genetic counselors, and laboratory scientists, as well as with patients and family members, will be enhanced through better communication support and information support. Health outcomes may be improved as a result of growing the capacity of the genetic services system through technology implementation.

\section{DISCUSSION}

The findings from our stakeholder study in the New Zealand genetic services delivery system have highlighted challenges surrounding medical genetic data sharing and usage. We have suggested some IT opportunities to better support human genetic variation KM. It is also essential to build innovations within an ethical and policy framework that will protect patients from genetic information misuse. 
Table 4 IT/KM innovation recommendations

\begin{tabular}{|c|c|}
\hline Technologies & Key functionalities for clinical genetic services \\
\hline DSS & $\begin{array}{l}\text { 1. To provide clinicians with genetic test utility information, e.g., on disease risk probability, prevalence, penetrance, and } \\
\text { cost/benefit of test. } \\
\text { 2. To assess individual patient's disease risk and identify patient/family at risk. } \\
\text { 3. To assist decision making as to test or not to test. } \\
\text { 4. To support referral process (according to protocols and possibly helped by e-Referral systems), e.g., for choosing a } \\
\text { service model and checking test availability. } \\
\text { 5. To collect right information in referrals, e.g., family history information. } \\
\text { 6. To manage patients in complying with clinical guidelines, e.g., drug dosage and surveillance. } \\
\text { 7. To check knowledge bases and flag patients for further intervention. }\end{array}$ \\
\hline e-Referrals & $\begin{array}{l}\text { 1. To support clinicians, genetic counselors, and laboratory scientists in streamlining consistent business processes of test } \\
\text { referral and referral triage. } \\
\text { 2. To track and share the status of referrals, appointments, and tests. } \\
\text { 3. To acknowledge referral receipts and to generate alerts if service level timeframes are not met. }\end{array}$ \\
\hline EHR/PHR & $\begin{array}{l}\text { 1. To securely store and share genetic test results. } \\
\text { 2. To align with patient-driven health care models by providing patients with the control over data sharing. } \\
\text { 3. To use role-based authorization in access control. }\end{array}$ \\
\hline $\begin{array}{l}\text { Data submission } \\
\text { tools }\end{array}$ & $\begin{array}{l}\text { 1. To systematically support database submission. } \\
\text { 2. To protect patients' right to privacy and confidentiality with proper data deidentification processes. }\end{array}$ \\
\hline $\begin{array}{l}\text { Other knowledge } \\
\text { processing } \\
\text { tools }\end{array}$ & $\begin{array}{l}\text { 1. To perform genetic data analysis. } \\
\text { 2. To search global knowledge bases. } \\
\text { 3. To store and manage local genetic data. } \\
\text { 4. To support workflow management. }\end{array}$ \\
\hline Ontologies & $\begin{array}{l}\text { 1. To control and standardize the use of terms and concepts, e.g., for interpreting the multiple names of gene (variants) } \\
\text { and unifying genome annotations. } \\
\text { 2. To represent genetic data in structure and semantics. } \\
\text { 3. To enable interoperability between information systems, e.g., by allowing electronic data transfer and search and } \\
\text { supporting API. }\end{array}$ \\
\hline $\begin{array}{l}\text { Knowledge } \\
\text { networking } \\
\text { tools }\end{array}$ & $\begin{array}{l}\text { 1. To facilitate communication and cooperation among health professionals. } \\
\text { 2. To enhance patients' contact with care givers, family members, and other patients, e.g., through online patient support } \\
\text { groups. }\end{array}$ \\
\hline
\end{tabular}

\section{Significance}

Medical genetic testing is starting to change clinical practice in many nations. This grounded study featured semistructured interviews with 48 participants spanning a wide range of stakeholders. Our research has identified several challenges for genetic information management. To realize the full potential of medical genetics knowledge for better health care outcomes, we need to overcome:

- The challenges of multiple service models.

- The inhibitors for genetic data sharing.

- The barriers to wider use of genetic testing.

We found four service delivery pathways in operation: the Patient-Doctor-Counselor Model, the Patient-Doctor-Lab Model, the Patient-Counselor-Lab Model, and the Patient-Lab (Commercial) Model. The lack of referral protocols and clinical guidelines to help clinicians choose among these models and manage patients after genetic tests presents a major barrier to their use of the genetic services. Divided attitudes are notable among our participants toward whether genomics is something requiring a different approach in health care delivery. The conflicting opinions regarding the nature and impact of genetic data are highlighted, especially their relevance to family members and their predictive power. The wider use of genetic testing technology is also impeded by the variable understanding of genetic test utilities and limited by the capacity of clinical genetic services. Taking a grounded theory approach, we identified that these factors are interrelated and relevant to stakeholder perception, attitude, utilization, and uptake of genetic testing technology and have inhibited the realization of the full potential of genetics knowledge. Given these constraints, the potential of human genetic variation knowledge in health care is on a "leash" at present. 
Targeting these problems, IT opportunities were discussed to support key tasks in genetic service delivery and to assist in appropriate use and access of genetic test results. Promising tools include DSS, e-Referrals, and EHR or PHR. Implementing these technologies and KM tools (e.g., data submission and other knowledge processing tools, ontology, and knowledge networking tools) may also improve knowledge processes in the domain and enhance knowledge networks.

The study results also present an imperative for health policy makers to promote open debates on ethical and legal issues entangling the use of genetic data. Establishment of effective ethical and policy frameworks is critical to protect patients, families, and health care professionals in leveraging the power of genetic information.

\section{Related work}

A closely related investigation in New Zealand was the 2003 GP survey regarding their knowledge and practice on genetic testing. ${ }^{13,48}$ This national survey found that GPs felt that they lacked experience and knowledge of genetic testing, especially on the appropriate terminology and procedures. It also reported that a substantial number of GPs are not sure how to access genetic advice for their patients. Our interviews have found little change regarding GP involvement in the genetic services delivery system. It seemed that clinicians, including GPs, need help to identify the utility of genetic tests.

There are a few studies, particularly in the United Kingdom, on cancer genetics services. ${ }^{15,16,49-52}$ These studies have highlighted the lack of knowledge in health professionals and the urgent need to develop their core competencies for genetic services delivery. They also pointed out the lack of communication and cooperation between genetics centers, and the primary, secondary, and tertiary health care providers. We have identified similar problems in the New Zealand context. However, the multiple service models we found differ from the single Patient-Doctor-Counselor Model in the United Kingdom. With multiple models in operation, clinicians need clear protocols for referral processes and clinical guidelines for managing patients.

Relevant work has also been conducted in the United States. The Department of Health and Human Services' Personalized Health Care Initiative is creating a foundation on which IT that supports personalized, predictive, and preemptive health care can be built. ${ }^{53}$ The Personalized Health Care Workgroup of the American Health Information Community is developing recommendations to facilitate data capture, storage, transmission, and use in clinical decision support, taking into account the issues related to standard reporting and incorporation of medical genetic or genomic tests and family health history data in the EHR. ${ }^{53}$ A 2009 study also promoted inclusion of genetic information in the EHR and highlighted the need to adopt sound privacy and security policies based on existing legislation, such as Genetic Information Nondiscrimination Act. ${ }^{54}$ Both topics emerged from our interviews as well. The promising technologies identified in our study include DSS, e-Referrals, EHR or PHR, and KM tools such as gene ontologies. Our grounded theory approach with multiple stakeholders addressed the fundamental debates surrounding what has been inhibiting the uptake and understanding of genetic testing technology in clinical practice.

In 2004, a survey of 1000 members of the Swedish general public found that many people intended to have a genetic test; they also found that living in an affected group and having some kind of experience of a hereditary disease may lead to an even more positive attitude toward genetic testing. ${ }^{55}$ However, it seems in our study that the knowledge level in people among the New Zealand general public about genetic testing is rather low and the attitude might be less positive. Therefore, we urge education and communication on the utility of genetic testing and open debate on the ethical and legal issues surrounding genetic information management.

\section{Study limitation}

The main limitation of this study is that it is based on personal perspectives from individual experience, which might not represent accurately the entire New Zealand genetic services system. The qualitative methods used did not engage sufficient numbers of participants to create statistically significant data; and, in any event, we were not using random sampling of a population but, conversely, deliberately seeking individuals who enhance the diversity of our stakeholder group. To some extent, our small sample size is offset by the experience of genetic counselors and clinicians, who have frequent direct interactions with large numbers of patients. The methods of theoretical sampling and data saturation supported the research rigor of our systematic grounded theory approach. In addition, we used the quantitative HAL semantic space modeling technique as one of the data triangulation methods to validate the qualitative analysis results. We have presented the New Zealand genetic services as a case study, and although we have made some comparison to the broader set of literature and policies internationally, the extent to which the New Zealand experience may apply to other jurisdictions is left largely to the reader.

\section{Future directions}

As mentioned by several participants in this study, the rapid development of genetic testing technologies, such as next generation sequencing technology, ${ }^{38}$ will soon enable the sequencing of any patient's whole genome. In addition, new genetics knowledge is discovered in many scientific disciplines that may be applicable in health care settings, such as Nutrigenomics (which discovers gene-diet interactions that may enable rationally selecting foods for optimal health or reduced risk of chronic disease ${ }^{56}$ ). The next question is how far off are we from routine genetic testing for everyone, with effective management of the test results for preventive and personalized medicine?

The privacy concerns that were collected in our study reveal the danger or downside in "unleashing" the power of genetic information, especially considering its social, legal, ethical, and psychological impact, which needs to be explored and debated. Of particular concern is the topic of discrimination, as our study collected people's legitimate concerns over the lack of legal protection for patients against insurance discrimination. As such, there is need for further study into potential patient harms, including distinctive concerns of specific cultural groups (e.g., Māori in the New Zealand context). Moreover, the philosophical debate in the light of advanced human genetics science is another interesting and useful research direction. This evokes issues of determinism, eugenics, free will, social desirability, moral responsibility, and human rights. Illustrative of the philosophical issues are technologies such as in vitro fertilization and preimplantation genetic testing as practiced in modern medicine.

Strategic planning and framework development are needed to enable more widespread participation in the active use of genetic information among health care professionals and to achieve a degree of uniformity and interoperability among nations. Establishment of such strategies and frameworks may require more research into the technical, organizational, and social issues that will open up public debate on these topics. 


\section{CONCLUSION}

Medical genetics presents great potential for improving health care. Based on interviews of a range of stakeholders, however, it is clear that the power of genetic information is on a leash in the health system due to the challenges around managing genetic testing. The inhibitors to realizing the full potential of human genetic variation knowledge for better health care outcomes include the challenge of multiple service models, and the barriers to data sharing and to wider use of genetic testing. This grounded study has provided a better understanding of the New Zealand clinical genetic services system and identified the need and opportunity to unleash the power of genetics knowledge. ITs and KM tools show promise for supporting key tasks in genetic services delivery, improving knowledge processes in the domain, and enhancing knowledge networks. In addition, the establishment of effective ethical and policy frameworks is important to protect health care professionals, patients, and families in leveraging the power of genetic information for better health care outcomes.

\section{ACKNOWLEDGMENTS}

This study was conducted in a $\mathrm{PhD}$ project partially funded by a Health Informatics New Zealand (HINZ) study award. The authors also thank Associate Professor Alexei Drummond for his guidance on bioinformatics and Associate Professor Andrew Shelling for his support in the study.

\section{REFERENCES}

1. Engstrom JL, Sefton MG, Matheson JK, Healy KM. Genetic competencies essential for health care professionals in primary care. J Midwifery Womens Health 2005;50:177-183.

2. IT Perspectives Inc. NHS Genetic Service Information Systems Output Based Specification. UK National Health Service, 2005. Available at: http://www. ngrl.org.uk/Manchester/Pages/Downloads/ITP-GEN3-OBSv05.pdf. Accessed November 3, 2005.

3. US National Library of Medicine. What is genetic testing? The US National Library of Medicine (NLM) Genetics Home Reference $>$ Handbook > Genetic Testing, 2008. Available at: http://ghr.nlm.nih.gov/ handbook/testing/genetictesting. Accessed July 7, 2008.

4. Bell J. The new genetics: the new genetics in clinical practice. BMJ 1998; 316:618-620.

5. Zimmern RL. The human genome project: a false dawn? Interview by Judy Jones. BMJ 1999;319:1282.

6. Bhandari M, Garg R, Glassman R, Ma PC, Zemmel RW. A genetic revolution in healthcare. Hosp $O$ 2000;3:58-62.

7. Glasner P. Beyond the genome: reconstituting the new genetics. New Genet Soc 2002;21:267-277.

8. McGuire AL, Fisher R, Cusenza P, et al. Confidentiality, privacy, and security of genetic and genomic test information in electronic health records: points to consider. Genet Med 2008;10:495-499.

9. Gene Tests. Educational materials: uses of genetic testing. Medical Genetics Information Resource (database online), 2004. Copyright, University of Washington, Seattle. 1993-2006. Available at: http://genetests.org/ servlet $/$ access $? \mathrm{id}=8888891 \& \mathrm{key}=\mathrm{ypo} 8 \mathrm{wK} 00 \mathrm{cjJaW} \& \mathrm{fcn}=\mathrm{y} \& \mathrm{fw}=\mathrm{GxFP} \&$ filename $=/$ concepts/primer/primerusesof.html. Accessed June 6, 2008.

10. Genetic Tools. Using genetic tests. Genetic tools: genetics through a primary care lens, 2005. Available at: http://www.genetests.org/servlet/ access? $\mathrm{id}=8888892 \& \mathrm{key}=3 \mathrm{JZ} \operatorname{og} 3 \mathrm{~s} \mathrm{X}$-tbDE $\& \mathrm{fcn}=\mathrm{y} \& \mathrm{fw}=$ OsE6 \&filename $=/$ tools/concepts/usingTests.html. Accessed June 6, 2008.

11. Personalized medicine coalition. The case for personalized medicine, 2006 Available at: http://www.personalizedmedicinecoalition.org/communications/ TheCaseforPersonalizedMedicine_11_13.pdf. Accessed July 7, 2008.

12. Calnan M, Wainwright D, Glasner P, Newbury-Ecob R, Ferlie E. 'Medicine's next goldmine?' The implications of new genetic health technologies for the health service. Med Health Care Philos 2006;9:33-41.

13. White S, McLeod D. Genetic testing: a survey of New Zealand general practitioners' knowledge and current practice, 2003. A report to the National Health Committee. Department of General Practice, Wellington School of Medicine. March 2003. Available at: http://www.nhc.health.govt.nz/moh.nsf/ pagescm/788/\$File/GeneticTestingFinalReport.pdf. Accessed March 3, 2009.

14. Suther S, Goodson P. Barriers to the provision of genetic services by primary care physicians: a systematic review of the literature. Genet Med 2003;5: $70-76$.
15. Wilson B. The challenge of developing evidence-based genetics health care in practice. Fam Cancer 2006;5:55-59.

16. Iredale R, Elwyn G, Edwards A, Gray J. Attitudes of genetic clinicians in Wales to the future development of cancer genetics services. $J$ Eval Clin Pract 2007; 13:86-89.

17. Metcalfe S, Hurworth R, Newstead J, Robins R. Needs assessment study of genetics education for general practitioners in Australia. Genet Med 2002; 4:71-77.

18. Glaser BG, Strauss AL. The discovery of grounded theory; strategies for qualitative research. Chicago, IL: Aldine Pub. Co., 1967.

19. Rhine J. The Grounded Theory Institute: the official site of Dr. Barney Glaser and Classic Grounded Theory, 2008. Available at: http://www. groundedtheory.com/. Accessed July 31, 2008.

20. QSR International Pty Ltd. NVIVO 8, 2008. Available at: http://www. qsrinternational.com/products nvivo.aspx. Accessed November 22, 2008.

21. Strauss AL, Corbin JM. Basics of qualitative research: techniques and procedures for developing grounded theory, 2nd ed. Thousand Oaks, CA: Sage Publications, 1998

22. Blaikie N. Approaches to social enquiry. Cambridge, MA: Polity, 1993

23. Thomas DR. A general inductive approach for analyzing qualitative evaluation data. Am J Eval 2006;27:237-246.

24. Mahoney J. Nominal, ordinal, and narrative appraisal in macrocausal analysis. AJS 1999;104:1154-1196.

25. Neuman WL. Social research methods: qualitative and quantitative approach, 5th ed. Boston, MA: Pearson Education, Inc., 2003.

26. Burgess C, Lund K. Representing abstract words and emotional connotation in a high-dimensional memory space. In: Shafto MG, Langley P, editors. Proceedings of the 19th Annual Conference of the Cognitive Science Society. Mahwah, NJ: Erlbaum, 1997:61-66.

27. Klein HK, Myers MD. A set of principles for conducting and evaluating interpretive field studies in information systems. MIS Ouart 1999;23:67-93.

28. Kock N. The three threats of action research: a discussion of methodological antidotes in the context of an information systems study. Decis Support Syst 2004;37:265-286.

29. Cohen T, Widdows D. Empirical distributional semantics: methods and biomedical applications. J Biomed Inform 2009;42:390-405

30. Chen G, Warren J, Riddle P. Semantic Space models for classification of consumer webpages on metadata attributes. J Biomed Inform 2010;43:725735

31. Agendia. MammaPrint gives you a clear answer in breast cancer prognosis, 2008. Available at: http://usa.agendia.com/en/mammaprint.html. Accessed February 2, 2009.

32. Genomic Health Inc. Oncotype DX breast cancer assay, 2009. Available at: http://www.oncotypedx.com/. Accessed February 2, 2009.

33. NIH-DOE Working Group on Ethical Legal and Social Implications of Human Genome Research. Genetic information and health insurance report of the task force on genetic information and insurance, 1993. Available at: http://www.genome.gov/10001750. Accessed June 6, 2008

34. US Congress. Genetic Information Nondiscrimination Act of 2008. Vol H.R 493, 2008. Washington DC: US Congress, 2008. Available at: http://www. ornl.gov/sci/techresources/Human_Genome/publicat/GINAMay2008.pdf. Accessed September 22, 2010.

35. Healthier Scotland Scottish Executive. Review of Genetics in relation to Healthcare in Scotland, 2006. Available at: www.scotland.gov.uk/Resource/ Doc/146336/0038294.pdf. Accessed March 3, 2009.

36. Biotechnology Australia. Genetics in family medicine: the Australian handbook for general practitioners, 2007. Available at: http://www.nhmrc. gov.au/your_health/egenetics/practitioners/gems.htm. Accessed September 22, 2010.

37. Hum Genet Society of Australasia. Direct to consumer genetic testing. Issue Paper, 2007. Available at: http://www.hgsa.com.au/images/UserFiles/ Attachments/DIRECTTOCONSUMERGENETICTESTING.pdf. Accessed February 2, 2009.

38. Mardis ER. Next-generation DNA sequencing methods. Annu Rev Genomics. Hum Genet 2008;9:387- 402.

39. Gu Y, Warren J. The diversity of genetic services delivery models in New Zealand. Health Care and Informatics Review Online 2009;13:28-37.

40. 23andme Inc. 23andMe genetics just got personal, 2008. Available at: https://www. 23andme.com/. Accessed November 22, 2008.

41. New Zealand Parliament. Human Rights Act 1993 No 82 (as at 01 October 2008), Public Act, 2008. Available at: http://www.legislation.govt.nz/act/ public/1993/0082/latest/DLM304475.html. Accessed June 6, 2010.

42. Human Rights Commission. Human rights in New Zealand today: New Zealand action plan for human rights, 2010. Available at: http://www.hrc. co.nz/report/chapters/chapter05/disabled02.html. Accessed June 6, 2010.

43. Gu Y, Warren J, Day K. Helping clinicians identify the clinical utility of genetic tests. Paper presented at the HISA Health Informatics Conference (HIC 2010). Melbourne, Australia, August 24-26, 2010.

44. New Zealand National Advisory Committee on Health and Disability. Molecular genetic testing in New Zealand. A report from the National Advisory Committee on Health and Disability (National Health Committee), 2003. 
Available at: http://www.nhc.health.govt.nz/moh.nsf/pagescm/665/\$File/ GeneticTestingNZ.pdf. Accessed January 18, 2010.

45. Fabian A. People born overseas. Statistics NZ, 1999. Available at: http:/ www.stats.govt.nz/products-and-services/Articles/born+ overseas-Oct99.htm. Accessed January 19, 2009.

46. New Zealand Department of Labour. Migration trends, 2008. Available at: http://www.immigration.govt.nz/NR/rdonlyres/814ACAD1-743B-4B8EA4FB-A2B492360FFD/0/MigrationTrends200607.pdf. Accessed November 22, 2008.

47. New Zealand Health Information Strategy Advisory Committee. eReferrals, 2009. Available at: http://www.hisac.govt.nz/moh.nsf/indexcm/hisac2-action zone-ereferrals. Accessed March 3, 2010

48. Morgan S, McLeod D, Kidd A, Langford B. Genetic testing in New Zealand: the role of the general practitioner. N Z Med J 2004;117:U1178.

49. Iredale R, Brain K, Gray J, France E. The information and support needs of women at high risk of familial breast and ovarian cancer: how can cancer genetic services give patients what they want? Fam Cancer 2003;2:119121.

50. Elwyn G, Edwards A, Iredale R, Davies P, Gray J. Identifying future models for delivering genetic services: a nominal group study in primary care. $B M C$ Fam Pract 2005;6:14.

51. Allen H, Maxwell L, Dibley N, et al. Patient perspectives on the Poole PCT cancer genetics service. Fam Cancer 2007;6:231-239.

52. Eeles R, Purland G, Maher J, Evans DG. Delivering cancer genetics services-new ways of working. Fam Cancer 2007;6:163-167.

53. Glaser J, Henley DE, Downing G, Brinner KM; Personalized Health Care Workgroup of the American Health Information Community. Advancing personalized health care through health information technology: an update from the American Health Information Community's Personalized Health Care Workgroup. J Am Med Inform Assoc 2008;15:391-396.

54. Sethi $\mathrm{P}$, Theodos K. Translational bioinformatics and healthcare informatics: computational and ethical challenges. Perspect Health Inf Manag 2009;6:1h.

55. Nordin K, Björk J, Berglund G. Factors influencing intention to obtain a genetic test for a hereditary disease in an affected group and in the general public. Prev Med 2004;39:1107-1114.

56. Ferguson LR. Nutrigenomics approaches to functional foods. $J$ Am Diet Assoc 2009; 109:452-458. 